\title{
Fuzzy Ensembles for Embedding Adaptive Behaviours in Semi-Autonomous Avatars in 3D Virtual Worlds
}

\author{
Julie Wall \\ Ebroul Izquierdo \\ Multimedia and Vision Research Group Multimedia and Vision Research Group Multimedia and Vision Research Group \\ Queen Mary, University of London \\ London, UK \\ julie.wall@eecs.qmul.ac.uk \\ Queen Mary, University of London \\ London, UK \\ ebroul.izquierdo@eecs.qmul.ac.uk \\ Qianni Zhang \\ Queen Mary, University of London \\ London, UK \\ qianni.zhang@eecs.qmul.ac.uk
}

\begin{abstract}
Semi-autonomous avatars should be both realistic and believable. The goal is to learn from and reproduce the behaviours of the user-controlled input to enable semi-autonomous avatars to plausibly interact with their human-controlled counterparts. A powerful tool for embedding autonomous behaviour is learning by imitation. Hence, in this paper an ensemble of fuzzy inference systems cluster the user input data to identify natural groupings within the data to describe the users movement and actions in a more abstract way. Multiple clustering algorithms are investigated along with a neuro-fuzzy classifier; and an ensemble of fuzzy systems are evaluated.

Index Terms-semi-autonomous avatar; fuzzy inference system; fuzzy ensembles; virtual environments;
\end{abstract}

\section{INTRODUCTION}

The emergence of ever more complex virtual worlds has led to a range of avatar embodiments which must interact with the world and other virtual characters in a believable and realistic manner. Traditionally, avatars have performed human-controlled actions in a passive manner. Conversely, autonomous agents are embedded with intelligent capabilities which enable them to act independently [1]. Midway between a passive avatar and an autonomous agent is a semiautonomous avatar, i.e. a user-controlled avatar which has been enriched with some autonomous behaviour to allow the avatar to act independently in certain scenarios [2]. This semiautonomous behaviour needs to be visually indistinguishable by emulating the behaviour and conduct of the human user and their means of controlling their avatar. This is achieved by applying intelligent techniques to learn from the user's behaviour in response to their actions with different objects and characters in the virtual world. This learned behaviour provides a powerful tool for the development of a control system for semi-autonomous behaviour which is realised when the avatar acts independently of the user.

Research on combining user input with autonomous behaviour to produce the appropriate behaviour for an avatar is a continual topic of interest [3]. This merging of user-driven and autonomous control takes many forms in the literature including the integration of autonomy with some form of directablity at the motivational, task and direct motor level
[4]; an autonomous agent control system which can choose to accept or reject an order from the user based on trust [5]; and crowd control simulations with differing levels of control: programmed, autonomous or guided [6], to highlight but a few. This is a vast research area where semi-autonomous behaviour has been employed for expressive behaviour, navigation, nonverbal emotional behaviour, avatar personalisation and many more applications [3].

This paper presents preliminary research in the development of an artificial intelligence control system which will enable a virtual semi-autonomous avatar to respond appropriately in real time to its complex, multi-faceted 3D environment. Upon interaction with objects in the world, complex autonomous behaviour will be exhibited without unnecessary time-consuming input from the user. The overall objective of this research is to record and learn the control actions from the user-controlled avatar and use these learned actions to enable an avatar to behave autonomously in a way that is representative of the user, e.g. if the user unexpectedly leaves the virtual world (due to connection loss), the autonomous behaviour will stand-in for the user.

In the proposed scenario presented in this paper, the setting of the 3D virtual world is a rural scene populated by the user-controlled avatar. Active behaviour consists of walking or running towards a set destination, i.e. around a circuitous track and interacting with different environmental objects along the way, such as stopping to open treasure chests or jumping over hurdles. This paper proposes a control system to enable a semi-autonomous avatar to decide which behaviour to depict through the use of fuzzy logic theory. Firstly, all of the user's movements and interactions with objects and other avatars will be recorded into a dataset. Distinct active behaviours are processed by separate fuzzy inference systems, each describing the users movements and actions in a more abstract way with specific objects of the world. Multiple fuzzy logic systems are investigated, grid partitioning, subtractive clustering, fuzzy c-means clustering (FCM) and neuro fuzzy systems; to determine which technique is the most suitable for working with the complex data of dynamic 3D virtual 


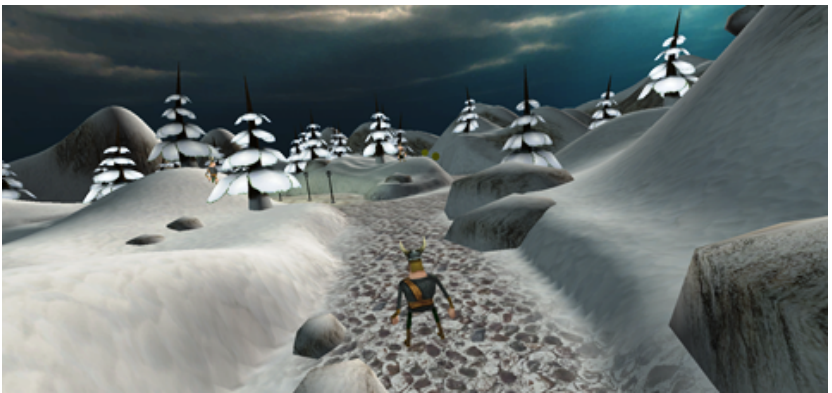

Fig. 1: 3D virtual world created using Unity

worlds in real time. Further examinations are carried out to determine how the trained fuzzy systems for individual events cooperate as an ensemble to fully describe the user's behaviour as they interact with the world as a whole. Ensembles have been used in cases where a single system trained with a large dataset is not practical; the data is divided and processed by different classifiers [7]. As a rule, combining intelligent systems improves both robustness and accuracy [8]. In our present prototype, the integrated development environment (IDE) for developing the 3D virtual world and semi-autonomous avatar is Unity3D, Matlab is used to process the event data and produce the ensemble of fuzzy inference systems.

Section 2 outlines the recording of the user-directed data. Section 3 introduces the fuzzy logic theory and provides a discussion of the four key fuzzy inferencing techniques in the literature; Section 4 describes the experiments carried out to determine the most suitable configuration for the fuzzy systems. Section 5 evaluates the fuzzy inference systems as they cooperate as an ensemble, while Section 6 concludes the paper.

\section{Event Detection AND Recording}

This section describes the recording of the observations of the semi-autonomous avatar as they interact with their 3D virtual environment. The types of events that are logged include descriptors for objects in the environment; position of the virtual character; distance of the virtual character to other virtual characters or environmental objects of importance; the means of interacting with the environment and other virtual characters, i.e. the specific behaviours in action; the reasons for the occurrence of these interactions, etc. The logged data is essentially a record of the users' behaviour over time. The more time the user spends navigating the world, the more data that will be compiled, and hence the subsequent control system will more accurately reflect the user's behaviour.

The 3D virtual environment was developed using Unity3D, see Figure 1. 3D model assets, many of which were freely available from the Unity3D asset store, were imported into the environment. The fully-rigged virtual character has seven different applied animations (behaviours) for interacting with the environment: walk, idle, fall, run, jump, shuffle and land. It is planned that future research will include the use of motioncapture to enable the application of hundreds of animations.

\section{FUZZY INFERENCE SySTEMS}

The indefinite quantity and complexity of the recorded data is converted into an ensemble of fuzzy inference systems. These will process the expected behaviours of the semiautonomous avatar rather than simply trying to mimic or copy the user-controlled character in every way. The fuzzy inference system is a logic computing structure composed of fuzzy set theory, fuzzy if-then rules and fuzzy reasoning [9]. There are numerous rationales for using fuzzy logic: it can be used to obtain a reasonable model when real world data is too complex to be utilised in a dynamic way in real time; it will provide a degree of uncertainty which will result in more believable and realistic actions for the semi-autonomous avatar; and fuzzy logic can control behaviours at run-time speeds and meet the requirements of real-time 3D simulations [10].

In order to produce the ensemble of fuzzy inference systems, the user input data must be clustered to identify natural groupings within the data which will enable a succinct embodiment of the system's behaviour to be modelled [11]. By partitioning the data into fuzzy subsets or clusters based on similarities within the data, the output can be predicted given the input [12]. There are many different algorithms in the literature for constructing a fuzzy inference system, however in this paper we will investigate the following four techniques:

Grid Partitioning: A simple, non-clustering Sugeno-based method which generates a fuzzy inference system with few membership functions per input [13]. However, the amount of rules increases exponentially with inputs as the rules describe all possible combinations of membership functions [14]. This method can suffer from the 'the curse of dimensionality' where there are too many rules for any practical learning algorithm to deal with, e.g. a fuzzy inference system with ten inputs that each have two membership functions will have $2^{10}$ (1024) rules. Grid partitioning is mainly used to provide the initial conditions for posterior neuro-fuzzy training.

Subtractive Clustering: A Sugeno-based method which generates a fuzzy inference system with a precise set of rules that model the data behaviour from complex input membership functions [13]. It is a fast one-pass method which estimates both the number of clusters and the cluster centres in a dataset [11].

Fuzzy C-Means Clustering (FCM): FCM produces a set of rules which describe the behaviour of the data where every input data point belongs to every cluster to some degree [14]; the extent of involvement to each cluster is specified by the membership grade. Essentially, FCM is an iterative optimization algorithm that aims to minimise the cost function $J$, it converges to a local minimum or a saddle point of the cost function [11] [15]:

$$
J=\sum_{k=1}^{n} \sum_{i=1}^{c} \mu_{i k}^{m}\left\|\chi_{k}-\nu_{i}\right\|^{2}
$$

where $n$ is the number of data points, $c$ is the number of clusters, $\mu_{i k}$ is the degree of membership of the $k$ th data point to the $i$ th cluster, $m$ is a constant greater than $1, \chi_{k}$ is the 
$k$ th data point and $\nu_{i}$ is the $i$ th cluster center. The degree of membership $\mu_{i k}$ is defined by:

$$
\mu_{i k}=\frac{1}{\sum_{j=1}^{c}\left(\frac{\left\|\chi_{k}-\nu_{i}\right\|}{\left\|\chi_{k}-\nu_{j}\right\|}\right)^{2 / m-1}}
$$

Neuro Fuzzy Systems: Neuro fuzzy hybrid systems are connectionist representations of fuzzy logic systems that harness the learning capabilities of various artificial neural network architectures for the tuning of membership functions or the configuration of rule bases. Arguably, the most famous neuro fuzzy is the Adaptive Neuro Fuzzy Inference System (ANFIS) [16]. ANFIS uses a hybrid learning algorithm to identify the membership function parameters of a single output, Sugenotype fuzzy inference system.

\section{EXPERIMENTAl EVAluation AND COMPARATIVE ANALYSIS}

Inputs to the fuzzy inference systems comprise information about the interaction between the user-controlled avatar and objects in the environment over time, i.e. for every time-step the following information will be logged: position and orientation in the 3D space with Cartesian coordinates, i.e. $x, y$ and $z$ coordinates; distance between the avatar and object; and event information such as a door opens when the avatar attempts to pass through or a coin disappears as the avatar collects it through jumping to make contact with it. Furthermore, for the semi-autonomous avatar to engage with human-controlled, semi-autonomous or completely autonomous avatars in a credible manner, it is very important that the initiation of contact, ongoing contact and the termination of contact appears lifelike, smooth and familiar [17]. Therefore, the distance between the avatar and the objects is also important as each stage of interaction is dependent on this.

The ensemble of fuzzy logic systems are comprised of a set of membership functions that describe the behaviour of the avatar in response to a specific object. These rules will log both the decisive behaviour that was performed and the inputs leading to that behaviour, i.e. IF the teacher indicates that they want all their student's attention AND the semi-autonomous avatar is within hearing distance THEN the semi-autonomous avatar turns to face the teacher without requiring control input from the user.

For two scenarios, an ensemble of fuzzy inference systems were constructed using the following techniques: grid partitioning, subtractive clustering, ANFIS and FCM. The scenarios consist of interaction with particular objects in the world. The first object is a 'hurdle'; in the scenario the semiautonomous avatar runs towards the hurdle, jumps over it and then runs away from it. The second object is a 'treasurechest' of the type found in many computer games; in the scenario the semi-autonomous avatar approaches the chest and opens it, collects the contents, and closes the chest before moving away from it. The fuzzy inference systems which have been constructed based on these scenarios have no prior knowledge of the particular objects themselves; the fuzzy

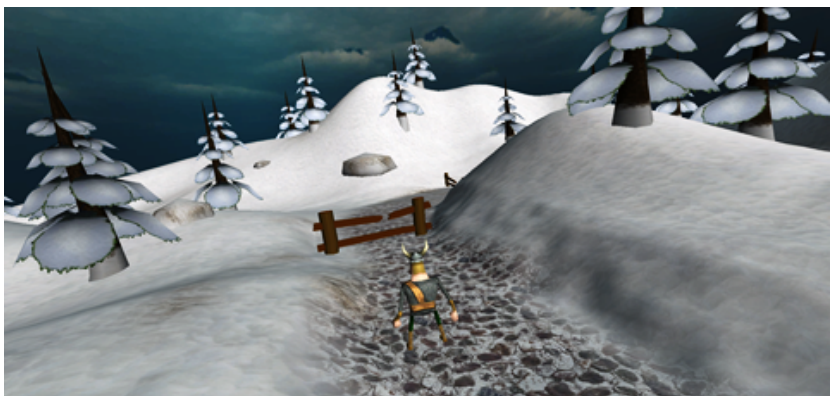

Fig. 2: One of the objects the avatar interacts with is a hurdle which the avatar must jump over

inference systems will rely solely on the interaction data recorded from the user-controlled avatar.

In the first scenario, see Figure 2, in which the avatar interacts with a hurdle, the dataset consists of three inputs and one output which will be used to train the fuzzy logic systems. The three inputs are the $x$ and $y$ positions of the avatar in the world and the distance from the selected object over time. The output data consists of the corresponding behaviours that the human-controlled avatar has exhibited, run, jump, walk, etc. For the purposes of testing the generalisation capabilities of the fuzzy logic systems, a second dataset was collected. This second dataset was only used to test the fuzzy logic systems once they had been trained. As the data is recorded in real time while the user is interacting with the virtual world, the number of elements in the datasets will vary with each run. In this first scenario, the training dataset consisted of 278 elements and the testing dataset consisted of 255 elements for each of the three inputs and one output. For both scenarios, the training and testing results are calculated using root mean squared error:

$$
R M S E=\sqrt{\frac{\sum_{i=1}^{n}\left(X_{o b s, i}-X_{\text {model }, i}\right)^{2}}{n}}
$$

where $X_{o b s}$ are the observed output values, $X_{\text {model }}$ are the estimated or modelled output values and $n$ are the number of instances.

In the second scenario, see Figure 3, in which the avatar interacts with a treasure chest, the dataset is more complex as it consists of four inputs and one output which will be used to train the fuzzy logic systems. The four inputs are the $x$ and $y$ positions of the avatar in the world, the distance from the selected object as before, and the state of the treasure chest is an added complication, i.e. the chest can have three states: open, inbetween and closed. Again, the output data consists of the corresponding behaviours that the human-controlled avatar has exhibited. As before, training and testing datasets were constructed; the training and testing datasets consist of 1368 and 1028 elements respectively for all inputs and the one output. There are more elements in the training and testing sets for scenario two as it takes longer for the user to make their way around the world while interacting with the chests, therefore more data is recorded. The remainder of this 


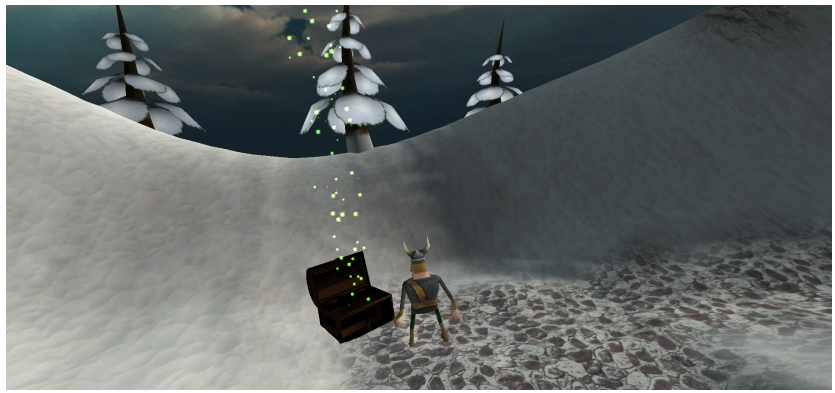

Fig. 3: The avatar also interacts with a chest which can be opened and closed

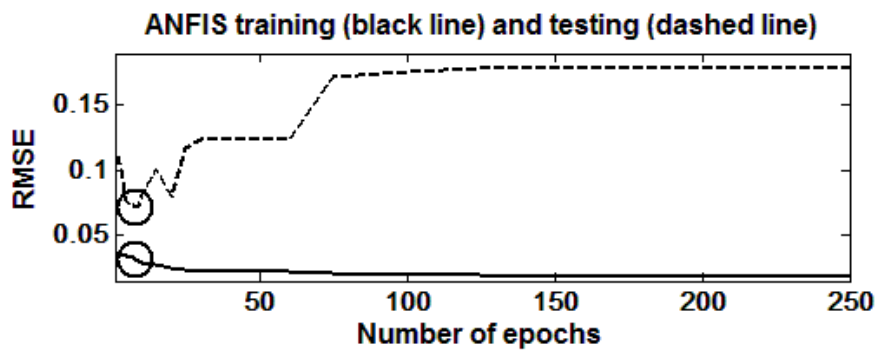

Fig. 4: Determining the optimal number of epochs for ANFIS training and testing. Subtractive clustering was used to provide the initial conditions.

section will outline a series of experiments which provide a comparison between the three clustering approaches and those same approaches when further tuned using ANFIS.

The experiments for generating a fuzzy inference system with grid partitioning and a neuro-fuzzy network with ANFIS consisted of applying the training data for differing numbers of membership functions. As the number of membership functions per input increases, the number of total rules also increases. However, regardless of the amount of partitions imposed by the grid, the training and testing results do not change. The same results are produced whether there are a large or a small number of partitions as the corresponding membership functions are inflexible for the real-valued complex data in this domain. Conversely, ANFIS can re-position the membership functions produced by grid paritioning, hence it can exploit the necessary degrees of freedom required to better represent the underlying pattern in the data. The main drawback of this approach, whether with or without ANFIS training, is the relatively large processing time required when compared with the other clustering techniques.

ANFIS is a training algorithm and as such, it is prone to overfitting. Consequently, it is important to determine when to stop training. This is done by evaluating the testing error as training proceeds to determine the optimal number of training epochs. As can be seen from Figure 4, even though the training error decreases as the number of epochs increases, the testing error shows generalisation is sub-optimal after eight epochs.

The experiments for subtractive clustering and the subsequent ANFIS network consisted of applying the training data
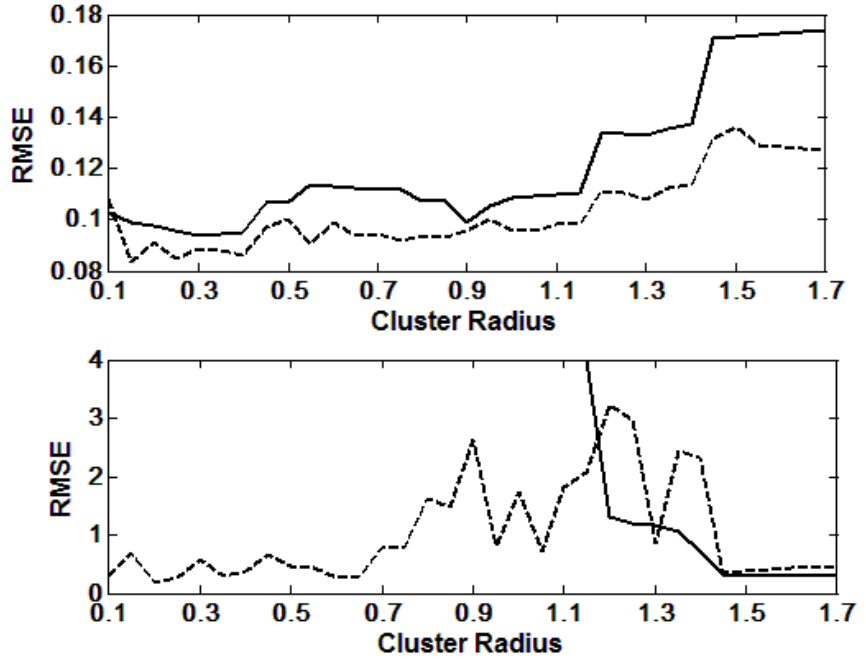

Fig. 5: Training (upper plot) and testing (lower plot) errors for the second scenario plotted against the number of clusters; the solid line plots the results for subtractive clustering, the dashed line plots the ANFIS results.

for different cluster radii, from a radius of 0.1 to 1.7 . As can be seen in Figure 5, for radius values greater than 1.7, ANFIS fails to generate any result as this produces an insufficient number of clusters for classification. As the radius values increase, the number of clusters decrease. As can be seen in the figure, ANFIS produces a smaller RMSE than a fuzzy inference system with subtractive clustering across all cluster radii. In fact, subtractive clustering fails to generalise for cluster radii less than 1.15, whereas ANFIS can generalise adequately for all cluster radii.

The final set of experiments compared the clustering ability of FCM in comparison to FCM with ANFIS. As expected, when ANFIS configured the arrangement of the FCM determined clusters, the overall training and testing error improved. Figure 6 demonstrates this improvement for all numbers of clusters in the training set.

Across both scenarios, it is clear from the results presented in Table I and Table II that the optimum configuration for developing a fuzzy inference system is to use FCM to generate the initial positions of the membership functions and to use ANFIS to further tune the system. Although the training and testing results using subtractive clustering and subtractive clustering with ANFIS for the first scenario produce a very small error, it is interesting to note that the amount of membership functions required is 244 while there are only 278 data points in the training data set. This produces a complex fuzzy inference system that takes a relatively long time to train in comparison to FCM with ANFIS.

\section{FuzZy Ensemble Evaluation}

The last section highlighted the generalisation capabilities of ANFIS within a particular scenario. However, each fuzzy system will only describe the behaviour of the user-controlled 


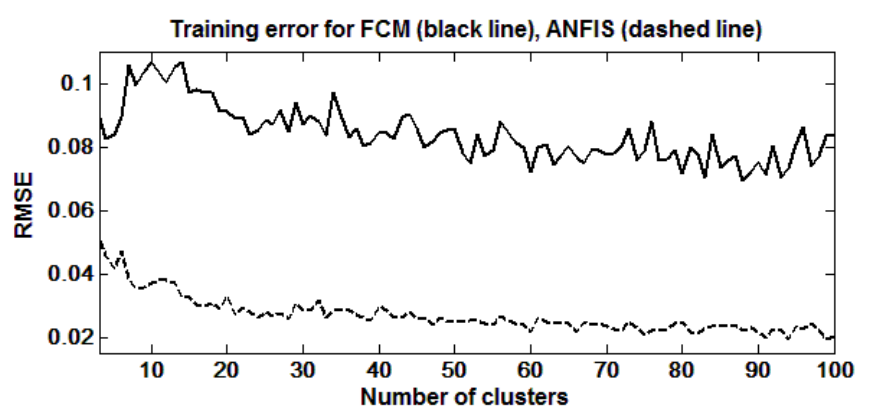

TABLE II: Training and testing results for the second scenario

\begin{tabular}{ccccc}
\hline \hline FIS Type & Training Error & Testing Error & Epochs & Clusters \\
\hline Grid Par. & 0.4600 & 0.5340 & - & 24 \\
ANFIS & 0.0911 & 0.2737 & 250 & 24 \\
\hline Sub. Clust. & 0.0979 & - & - & 13 \\
ANFIS & 0.0909 & 0.1291 & 700 & 13 \\
\hline FCM & 0.2704 & 0.3250 & - & 6 \\
ANFIS & 0.0973 & 0.2226 & 30 & 6 \\
\hline
\end{tabular}

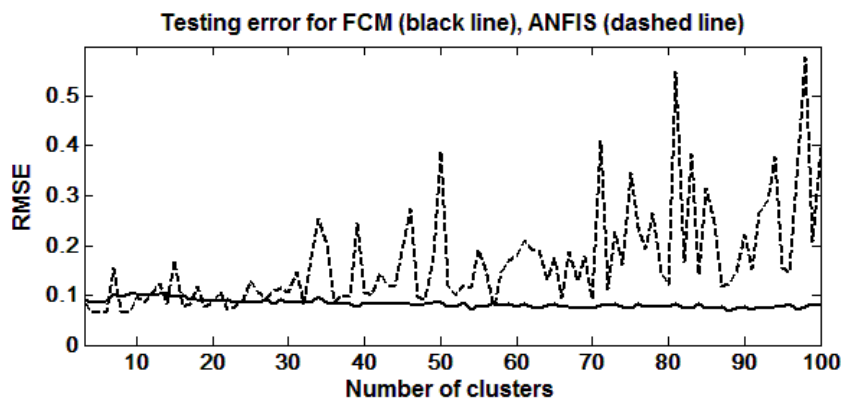

Fig. 6: Comparison between FCM and FCM with ANFIS, for the hurdle scenario.

TABLE I: Training and testing results for the first scenario

\begin{tabular}{ccccc}
\hline \hline FIS Type & Training Error & Testing Error & Epochs & Clusters \\
\hline Grid Par. & 0.9282 & 0.9535 & - & 8 \\
ANFIS & 0.0393 & 0.0620 & 45 & 8 \\
\hline Sub. Clust. & $5.63 e^{-16}$ & 0.0666 & - & 244 \\
ANFIS & $1.45 e^{-5}$ & 0.0064 & 8 & 244 \\
\hline FCM & 0.0729 & 0.0752 & - & 5 \\
ANFIS & 0.0398 & 0.0645 & 40 & 5 \\
\hline
\end{tabular}
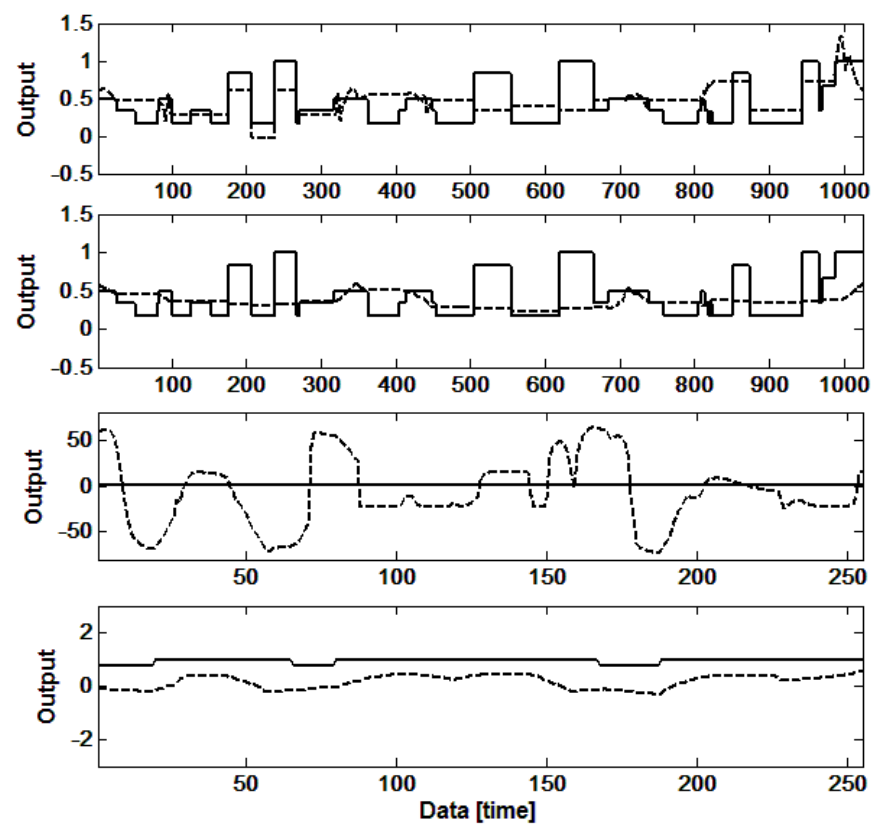

Fig. 7: Expected outputs (solid line), actual outputs (dashed line)

avatar as they interact with individual objects of the virtual world. To fully describe the user's behaviour as they interact with the world as a whole, i.e. to create a control system to enable the avatar to behave autonomously, each fuzzy system must cooperate as an ensemble. In this way, each member of the ensemble is presented with the users' control data for every possible scenario. The appropriate individual fuzzy inference system, as a part of the ensemble, will not only have to activate positively for its' own particular scenario it was designed for, it will also have to not adversely affect the ensemble output for the scenarios it was not designed for. Hence this section investigates the behaviour of the individual fuzzy inference systems developed for each specific scenario as they are grouped into an ensemble.

From the investigations of the different clustering algorithms and ANFIS for the individual fuzzy inference systems, it was clear that ANFIS provided the best approximation of the user's behaviour within the individual scenarios. However, it is commonly known that ANFIS, when presented with data that is substantially different to the data it was presented with during training, can oscillate due to its excess of degrees of freedom [18]. Conversely, a fuzzy inference system developed

using FCM has less degrees of freeedom and thus is better behaved in a similar situation.

Figure 7 highlights the issues a fuzzy inference system configured using ANFIS has when presented with data from another scenario. In the figure, subplots 1 and 2 show how the FCM with ANFIS and FCM fuzzy systems trained for the chest scenario perform when presented with data from the same scenario. It is clear that the fuzzy system optimised with ANFIS performs better. However, in subplot 3, the fuzzy system optimised by ANFIS produces an output that is highly unstable when presented with data from an unfamiliar scenario. In comparison, the last subplot, shows how an FCM fuzzy system without ANFIS can produce an output which does not adversely affect the overall ensemble when also presented with unfamiliar data. Furthermore, the actual RMSE results quantify these conclusions as can be seen in Table III.

\section{CONCLUSION}

This paper presented experimental results in the development of an AI framework which will embed semi-autonomous behaviour into an avatar which also takes direction from a human user. Four fuzzy inferencing techniques were investigated and compared to determine their abilities to process 
TABLE III: Actual and expected outputs from FCM and FCM with ANFIS fuzzy systems

\begin{tabular}{ccc}
\hline \hline FIS Type & Input Data & Testing Error (RMSE) \\
\hline FCM with ANFIS. & Chest data & 0.2814 \\
FCM & Chest data & 0.3250 \\
FCM with ANFIS. & Hurdle data & 37.7259 \\
FCM & Hurdle data & 0.8149 \\
\hline
\end{tabular}

the complex data of dynamic 3D virtual worlds in real time. An ensemble of these fuzzy systems was evaluated to fully describe the user's behaviour as they interact with the world as a whole. It is planned to extend this work to include a classifier which will determine which behaviour the semiautonomous avatar will portray from conflicting outputs from the ensemble of fuzzy inference systems. From the comparison of fuzzy systems and the evaluation of the ensemble of fuzzy systems presented in this paper, FCM clustering will provide membership functions for the individual members of the fuzzy ensembles and will be employed for this extended work due to its advantages of rapid processing of the complex data sets and its ability to provide consistent generalisation and successful classification.

\section{ACKNOWLEDGMENT}

This work was supported by the EU funded IP project REVERIE under contract FP-287723.

\section{REFERENCES}

[1] S. Franklin and A. Graesser, "Is it an agent, or just a program?: A taxonomy for autonomous agents," Intelligent Agents III Agent Theories, Architectures, and Languages, pp. 21-35, 1997.

[2] M. Gillies and D. Ballin, "Integrating autonomous behavior and user control for believable agents," in Proceedings of the Third International Joint Conference on Autonomous Agents and Multi-agent Systems, IEEE, 2004, vol. I, pp. 336-343.

[3] M. Gillies, D. Ballin, and N.A. Dodgson, "Semi-autonomous avatars: A new direction for expressive user embodiment," Animating expressive characters for social interaction, vol. 74. pp. 235-255, John Benjamins Publishing Company, 2008.

[4] B.M. Blumberg and T.A. Galyean, "Multi-level direction of autonomous creatures for real-time virtual environments," in Proceedings of the 22nd annual conference on Computer Graphics and Interactive Techniques, ACM, 1995, pp. 47-54.

[5] A. Caicedo and D. Thalmann, "Virtual humanoids: Let them be autonomous without losing control," in The Fourth International Conference on Computer Graphics and Artificial Intelligence, 2000.

[6] S.R. Musse, F. Garat, and D. Thalmann, "Guiding and interacting with virtual crowds in real-time," in Proceedings of Workshop Eurographics Computer Animation and Simulation, 1999.

[7] P. Baraldi, R. Razavi-Far and E. Zio, "Bagged ensemble of Fuzzy CMeans classifiers for nuclear transient identification," Annals of Nuclear Energy, vol. 38, no. 5, pp. 1161-1171, 2011.

[8] M. Korytkowski, R. Nowicki, L., Rutkowski and R. Scherer, "Merging ensemble of neuro-fuzzy systems," International Conference on Fuzzy Systems, IEEE, 2006, pp. 1957-1957.

[9] J.S.R. Jang, "Fuzzy inference systems," Upper Saddle River, NJ: PrenticeHall, 1997.

[10] R.M. Aguilar, V. Muoz, and Y. Callero, "Control application using fuzzy logic: Design of a fuzzy temperature controller," Fuzzy Inference System - Theory and Applications, Dr. Mohammad Fazle Azeem (Ed.), ISBN: 978-953-51-0525-1, pp. 379-396, May 2012.

[11] S.L. Chiu, "Fuzzy model identification based on cluster estimation," Journal of Intelligent and Fuzzy systems, vol. 2, no. 3, pp. 267-278, 1994.
[12] T.J. Ross, "Automated methods for fuzzy systems," Fuzzy Logic with Engineering Applications, Second Edition, John Wiley \& Sons, Ltd., 2004.

[13] D. Kaur and D. Baumgartner, "A comparative analysis of neuro-fuzzy and grammatical evolution models for simulating field-effect transistors," in WRI World Congress on Computer Science and Information Engineering, IEEE, 2009, vol. 5, pp. 179-183.

[14] A.H. Mesbahi, D. Semnani, and S.N. Khorasani, "Performance prediction of a specific wear rate in epoxy nanocomposites with various composition content of polytetrafluoroethylen (PTFE), graphite, short carbon fibers (CF) and nano- $\mathrm{TiO}_{2}$ using adaptive neuro-fuzzy inference system (ANFIS)," Composites Part B: Engineering, 2011.

[15] J.C. Bezdek, R.J. Hathaway, M.J. Sabin, and W.T. Tucker, "Convergence theory for fuzzy c-means: Counter-examples and repairs," IEEE Transactions on Systems, Man, and Cybernetics, vol. 17, no. 5, pp. 873-877, 1987.

[16] J. Jang, "ANFIS: Adaptive-network-based fuzzy inference system," IEEE Transactions on Systems, Man, and Cybernetics, vol. 23, no. 3, pp. 665686, 1993.

[17] C.L. Sidner, C. Lee, C.D. Kidd, N. Lesh, and C. Rich, "Explorations in engagement for humans and robots," Artificial Intelligence, Elsevier, vol. 166, no. 12, pp. 140164, 2005.

[18] C.E. Carrano, R.H.C. Takahashi, W.M. Caminhas and O.M. Neto, "A genetic algorithm for multiobjective training of ANFIS fuzzy networks," Evolutionary Computation, World Congress on Computational Intelligence, IEEE, 2008, pp. 3259-3265. 